\title{
THE CLASSIFICATION PROBLEM FOR AUTOMORPHISMS OF C*-ALGEBRAS
}

\author{
MARTINO LUPINI
}

\begin{abstract}
We present an overview of the recent developments in the study of the classification problem for automorphisms of $\mathrm{C}^{*}$-algebras from the perspective of Borel complexity theory.
\end{abstract}

\section{CONTENTS}

1. Introduction

2. $\mathrm{C}^{*}$-algebras and noncommutative dynamics

(1)

4. Conjugacy 10

5. Unitary equivalence $\quad 12$

6. Cocycle conjugacy 14

References $\quad 17$

\section{INTRODUCTION}

Borel complexity theory is an area of logic that studies, generally speaking, the relative complexity of classification problems in mathematics. The main ideas and methods for such a study come from descriptive set theory, which can be described as the analysis of definable sets and their properties. In the framework of Borel complexity theory, a classification problem is regarded as an equivalence relation on a Polish space. Perhaps after a suitable parametrization, this virtually covers almost all concrete classification problems in mathematics.

Under the assumption that the classes of objects under consideration are naturally parametrized by the points of a Polish space, it is to be expected - and demanded - that a satisfactory classification satisfy some constructibility assumption. A sensible notion of constructibility is to be Borel measurable with respect to the given parametrizations. This leads to the following definition, first introduced and studied in [33]. Suppose that $E, E^{\prime}$ are equivalence relations on standard Borel spaces $X, X^{\prime}$. A Borel reduction from $E$ to $E^{\prime}$ is a Borel function $f: X \rightarrow X^{\prime}$ such that

$$
f\left(x_{1}\right) E^{\prime} f\left(x_{2}\right) \quad \text { if and only if } x_{1} E x_{2}
$$

2000 Mathematics Subject Classification. Primary 03E15, 46L40; Secondary 46L35, 46L57.

Key words and phrases. C*-algebra, automorphism, Borel complexity, Borel reduction, turbulence, Cuntz algebra.

The author was supported by the York University Susan Mann Dissertation Scholarship. 
for every $x_{1}, x_{2} \in X$. The function $f$ can be seen as a constructive way to assign to the objects of $X$ complete invariants up to $E$ that are $E^{\prime}$-classes. Equivalently a Borel reduction can be seen as an embedding of the quotient space $X / E$ into the quotient space $X^{\prime} / E^{\prime}$ that is "definable", in the sense that it admits a Borel lifting from $X$ to $X^{\prime}$.

The notion of Borel reduction allows one to compare the complexity of different classification problems. If there exists a Borel reduction from $E$ to $E^{\prime}$, then $E$ is Borel reducible to $E^{\prime}$. Such a notion formalizes the assertion that $E^{\prime}$ is at least as complicated as $E$ or, alternatively, that $E$ is at most as complicated as $E^{\prime}$. In fact any Borel assignment of complete invariants up to $E^{\prime}$ yields a Borel assignment of complete invariants up to $E$ by precomposing with a Borel reduction. Therefore if one knows how to classify the objects of $X^{\prime}$ up to $E^{\prime}$, then one also knows via the Borel reduction how to classify the objects of $X$ up to $E$.

Borel reducibility gives a way to compare the complexity of classification problems. Some canonical equivalence relations are then used as benchmarks of complexity. This allows one to build a hierarchy of classification problems in mathematics. The first natural benchmark is provided by the relation $=_{\mathbb{R}}$ of equality of real numbers. An equivalence relation $E$ is smooth if it is Borel reducible to $=\mathbb{R}$. One can replace $\mathbb{R}$ with any uncountable standard Borel space [49, §15.6]. Smoothness amounts at saying that one can (explicitly) parametrize the equivalence classes of $E$ by the points of a standard Borel space. While the most satisfactory classification results in mathematics are of this form, it was soon realized that many natural and interesting equivalence relations are not smooth. This observation goes back to the work of Mackey and Glimm on irreducible representations of groups and $\mathrm{C}^{*}$-algebras [38, 63].

One of the main goals of Borel complexity theory is to refine the analysis in the nonsmooth case, and to understand if any satisfactory classification, possibly involving a more general kind of invariants, is possible. A more generous notion than smoothness is being classifiable by countable structures. In this case the benchmark of complexity is provided by the relation $\cong_{\mathcal{L}}$ of isomorphism of $\mathcal{L}$-structures for some first-order language $\mathcal{L}$. An equivalence relation is then classifiable by countable structures if it is Borel reducible to $\cong_{\mathcal{L}}$ for some first-order language $\mathcal{L}$.

Assuming without loss of generality that $\mathcal{L}$ is a relational language, the space of $\mathcal{L}$-structures can be regarded as the space $\prod_{R} 2^{\mathbb{N}^{n} R}$ where $R$ ranges over the symbols in $\mathcal{L}$ and $n_{R}$ denotes the arity of $R$. In this parametrization the relation of isomorphism coincides with the orbit equivalence relation of the canonical action of the group $S_{\infty}$ of permutations of $\mathbb{N}$. Conversely the orbit equivalence relation associated with a continuous action of $S_{\infty}$ on a Polish space can be regarded as the relation of isomorphism within some Borel class of $\mathcal{L}$-structures for a suitable language $\mathcal{L}$ [34, Theorem 3.6.1].

The natural next step beyond countable structures is to consider orbit equivalence relations of arbitrary Polish group actions. An equivalence relation is then classifiable by orbits if it is Borel reducible to the orbit equivalence relation associated with a Polish group action. In view of the López-Escobar theorem for continuous logic [6, 13], any orbit equivalence relation of a Polish group action can be identified with the relation of isomorphism within some Borel class of separable $\mathcal{L}$-structures for a language $\mathcal{L}$ in the logic for metric structures [5, 28]. Conversely any such a relation of isomorphism is Borel reducible to the action of a Polish group [23]. Beside classifiability by countable structures and by orbits, there are many other natural benchmarks of complexity, such as those corresponding to the classes of analytic equivalence relations that are hyperfinite, hypersmooth, treeable, or countable. 
The notion of Borel reducibility leads to a very rich and interesting theory, an account of which can be found in [34]. Albeit highly mixed and interlaced, one can isolate two main directions of research. One concerns the general study of the hierarchy of Borel (or analytic) equivalence relations under Borel reducibility. Remarkable examples of this approach are the many deep dichotomy results for Borel equivalence relations, starting from the seminal results of Silver [80] and Harrington-Kechris-Louveau [39]. A survey on this line of research can be found in [45, 50,65$]$.

The other direction aims at the study of important examples of equivalence relations coming from the practice of mathematics in various fields. The goal of this investigation is to determine as precisely as possible where these concrete classification problems sit in the Borel complexity hierarchy. Such an analysis is valuable in that it provides information on which classification results are possible, and what is the right kind of invariants to classify a given class of objects.

Beyond the smooth case, many explicit classification results in mathematics involve countable structures up to isomorphism as invariants. It is therefore of great importance to understand when an equivalence relation is classifiable by countable structure. Suppose that the equivalence relation under consideration is the orbit equivalence relation $E_{G}^{X}$ of a Polish group action $G \curvearrowright X$. The above description of isomorphism relations of countable structures as actions of $S_{\infty}$ suggest that one can use dynamical properties of the action $G \curvearrowright X$ to rule out the existence of a Borel reduction of $E_{G}^{X}$ to $E_{Y}^{S_{\infty}}$ for any $S_{\infty}$-space $Y$. For example it is well known that if there is a dense orbit, and all orbits are meager (a condition known as generic ergodicity) then the orbit equivalence relation $E_{G}^{X}$ is not smooth by [34, Proposition 6.1.10]. By locally strengthening this condition, Hjorth introduced the notion of (generically) turbulent action.

Suppose that $X$ is a Polish $G$-space, $x \in X, U$ is an open neighborhood of $x$ in $X$, and $V$ is an open neighborhood of the identity in $G$. The local orbit $\mathcal{O}(x, U, V)$ is the set of all points that can be reached from $x$ by applying elements of $V$ without ever leaving $U$. A point $x$ of $X$ is turbulent if for every nonempty open subset $U$ of $X$ and every open neighborhood $V$ of the identity of $G$ the local orbit $\mathcal{O}(x, U, V)$ is somewhere dense. The $G$-orbit of a turbulent points is a turbulent orbit.

Definition 1.1 (Hjorth, 2000). The action $G \curvearrowright X$ is turbulent if every orbit is dense, meager, and turbulent. It is generically turbulent if there is a $G$-invariant $G_{\delta}$ subset $C$ of $X$ such that the restriction of the action of $G$ to $C$ is turbulent.

Since the set of turbulent points is a $G$-invariant $G_{\delta}$ set, a $G$-space is generically ergodic if and only if every orbit is meager, and there is a dense turbulent orbit. The following is the main result of Hjorth's turbulence theory.

Theorem 1.2 (Hjorth, 2000). Suppose that $G \curvearrowright X$ is a generically turbulent Polish group action. If $Y$ is an $S_{\infty}$-space, and $f: X \rightarrow Y$ is a Baire measurable function that maps $G$-orbits into $S_{\infty}$-orbits, then there is an $S_{\infty}$-orbit whose preimage under $f$ is comeager. In particular $E_{G}^{X}$ is not classifiable by countable structures.

Hjorth's theory of turbulence is currently the main tool to establish results of nonclassifiability by countable structures. A more general approach in terms of forcing has been recently developed by Zapletal [87, 88].

Among the classifications problems that have been proved intractable by means of turbulence, there are unitary equivalence of irreducible representations of locally compact groups [41] and 
$\mathrm{C}^{*}$-algebras [25, 53], conjugacy of ergodic measure-preserving transformations of the standard probability space [32, 43], and unitary conjugacy of self-adjoint and unitary operators on the Hilbert space [52].

All the equivalence relations mentioned above are classifiable by the orbits of Polish group actions. Many naturally occurring classification problems have been shown to transcend such a benchmark, and in fact being of maximum complexity for all analytic equivalence relations. These relations that are complete for analytic equivalence relations include isomorphism and (complemented) biembeddability of Banach spaces, isomorphism of Polish groups, uniform homeomorphism of complete separable metric spaces [31], isomorphism of operator spaces [2], bi-embeddability of countable graphs [60].

Considering the importance that classification - in the form of the Elliott classification program [22, 24] - has in the modern theory of $\mathrm{C}^{*}$-algebras and $\mathrm{C}^{*}$-dynamics, it is not surprising that Borel complexity theory has provided interesting new information on the subject. The descriptive analysis of the classification problem for $\mathrm{C}^{*}$-algebra has been initiated in [12, 29, 30], and continued in [23, 76, 89]. In this survey we will focus on some new results on the Borel complexity of automorphisms of $\mathrm{C}^{*}$-algebras, mostly from the papers [36, 54, 62].

Acknowledgments. We would like to thank Ilijas Farah for his several comments on earlier versions of this survey that - we believe - significantly contributed to improve the presentation of the material. We are also grateful to Samuel Coskey for sharing his notes on the relation of isomorphism of countable torsion-free abelian groups.

\section{2. $\mathrm{C}^{*}$-ALGEBRAS AND NONCOMMUTATIVE DYNAMiCS}

An (abstract) $C^{*}$-algebra $A$ is a complex algebra endowed with a norm and an involution $x \mapsto x^{*}$ satisfying $\|x y\| \leq\|x\|\|y\|$ and the $\mathrm{C}^{*}$-identity $\left\|x^{*} x\right\|=\|x\|^{2}$ for $x, y \in A$. Suppose that $H$ is a Hilbert space. Let $B(H)$ be the algebra of bounded linear operators on $H$. Considering on $B(H)$ the operator norm and the involution that assigns to every operator its adjoint yields a $\mathrm{C}^{*}$-algebra structure on $B(H)$. A (concrete) $\mathrm{C}^{*}$-algebra is then a closed subalgebra of $B(H)$ that is self-adjoint, i.e. closed under taking adjoints. The foundational result of the theory of $\mathrm{C}^{*}$-algebra due to Gelfand and Naimark asserts that any abstract $\mathrm{C}^{*}$-algebra is isomorphic to a concrete one [37].

For simplicity we will assume all $C^{*}$-algebras to be unital, i.e. to contain a multiplicative identity 1 , and separable. These can be concretely identified with the separable closed self-adjoint subalgebras of $B(H)$ that contain the identity operator. There are many good references for the theory of $\mathrm{C}^{*}$-algebras [8, 11, 16]. In the following we will mostly refer to [8] for convenience.

The original motivation in the development of the theory of $\mathrm{C}^{*}$-algebras was to provide rigorous mathematical foundation to quantum mechanics [79]. In essence the "quantization process" consists in replacing functions with operators on the Hilbert space. If $K$ is a compact metrizable space, then the space $C(K)$ of complex-valued continuous functions on $K$ is a $\mathrm{C}^{*}$ algebra with respect to the pointwise operations and the uniform norm. The $\mathrm{C}^{*}$-algebras of this form are precisely the abelian ones [8, II.2.2.4]. Arbitrary $\mathrm{C}^{*}$-algebras can therefore be thought as quantized or noncommutative spaces. The theory of $\mathrm{C}^{*}$-algebras is thus sometimes referred to as noncommutative topology. The fact that these quantized spaces have no actual points is consistent with the principle that it is physically meaningless to speak of a "point" in the phase space of a quantum particle [19]. 
A similar quantization process can be applied to topological dynamical systems. Classically a topological dynamical system is given by a pair $(K, T)$ where $K$ is a compact metrizable space and $T$ is a homeomorphism of $K$. Dynamical systems can be thought as symmetries of a space, but also as specifying the (discrete) time evolution of a system. In order to understand what is the right quantized analog of dynamical systems, one can observe that a homeomorphism $T$ of $K$ canonically induces a map $\alpha_{T}: C(K) \rightarrow C(K)$ defined by $f \mapsto f \circ T$. Such a map is an automorphism of $C(K)$, in the sense that it preserves all the $\mathrm{C}^{*}$-algebra structure of $C(K)$. Conversely given any automorphism $\alpha$ of $C(K)$ there is a unique homeomorphism $T$ of $K$ that corresponds to $\alpha$ via the above relation. Therefore the quantized version of a topological dynamical system is a pair $(A, \alpha)$ where $A$ is a $\mathrm{C}^{*}$-algebra, and $\alpha$ is an automorphism of $A$. The study of automorphisms of $\mathrm{C}^{*}$-algebras can also be described as noncommutative topological dynamics.

The automorphisms of a $C^{*}$-algebra $A$ form a group Aut $(A)$ under composition. Such a group is endowed with a canonical Polish group topology, which is the weakest topology making the point-evaluations continuous. This observation opens up the possibility to the use of descriptive set-theoretic methods in the study of automorphisms of $\mathrm{C}^{*}$-algebras.

In the classical setting, topological dynamical systems are naturally studied and classified up to the relation of conjugacy. Two systems $T, T^{\prime}$ on the same space $K$ are conjugate if they are equal modulo a symmetry of the space. This means that there is an invertible continuous transformation $S$ of $K$ such that $S \circ T=T^{\prime} \circ S$. There is a straightforward quantized version of such a notion: two automorphisms $\alpha, \alpha^{\prime}$ of a $\mathrm{C}^{*}$-algebra $A$ are conjugate if there is an automorphism $\gamma$ of $A$ such that $\alpha \circ \gamma=\gamma \circ \alpha^{\prime}$. Equivalently, $\alpha$ and $\alpha^{\prime}$ are conjugate inside the automorphism group Aut $(A)$ of $A$. However this is not the only possible analog of conjugacy in the noncommutative setting. In order to define the other one, we need to introduce the important notion of unitary element.

An element $u$ of $A$ is unitary if it satisfies $u u^{*}=u^{*} u=1$. When $A$ is concretely represented as an algebras of operators on $H$, this is equivalent to the assertion that $u$ is a unitary operator on $H$, i.e. a surjective linear isometry. The unitary elements of $A$ form a multiplicative group $U(A)$ which is Polish when endowed with the norm topology. Any unitary element $u$ induces an automorphism Ad $(u)$ of $A$ defined by $x \mapsto u x u^{*}$. Automorphisms of this form are called inner and form a normal subgroup $\operatorname{Inn}(A)$ of Aut $(A)$. These are the automorphisms of $A$ that come from "rotating" the Hilbert space $H$ one which $A$ is represented, and can be regarded as trivial. It is therefore natural, in trying to classify the automorphisms of $A$, not to distinguish between automorphisms that are equal up to an inner automorphism. This leads to the notion of unitary equivalence of automorphisms of $A$.

Two automorphisms $\alpha, \beta$ of $A$ are unitarily equivalent if $\alpha=\operatorname{Ad}(u) \circ \beta$ for some unitary $u$ of $A$. Equivalently the images of $\alpha$ and $\beta$ in the quotient group $\operatorname{Aut}(A) / \operatorname{Inn}(A)$ are equal. Combining unitary equivalence with conjugacy leads to the definition of cocycle conjugacy. Two automorphisms are cocycle conjugate if one is unitarily equivalent to a conjugate of the other. Equivalently their images in the quotient group $\operatorname{Aut}(A) / \operatorname{Inn}(A)$ are conjugate. The name cocycle conjugacy comes from the fact that it can be described in terms of perturbations by a cocycle.

In the commutative setting, conjugacy and cocycle conjugacy coincide since there are no nontrivial inner automorphisms. In the noncommutative setting they behave in general very differently. It could be matter of debate which one is the "right" quantized analog of conjugacy 
of homeomorphisms. It is an empiric fact that most satisfactory classification results concern the relation of cocycle conjugacy. This is not a coincidence, as it has recently been confirmed in [54] by means of Hjorth's theory of turbulence that the relation of conjugacy is not well behaved for "most" $\mathrm{C}^{*}$-algebras.

Similar conclusions hold for the relation of unitary equivalence. In fact it has recently been shown in [62] that such an equivalence relation is not classifiable by countable structures whenever it is not smooth. Such a dichotomy is reminiscent of the analogous phenomenon for the relation of unitary equivalence of irreducible representations of $\mathrm{C}^{*}$-algebras. A classical result of Glimm asserts that such a relation is smooth precisely when $A$ is type I [38]. Recently Glimm's result has been refined by showing that in the non type I case the irreducible representations are not classifiable by countable structures [25, 41, 53].

The situation for the relation of cocycle conjugacy if far less clear. Many positive classification results have been obtained in special cases including, most notably, automorphisms of Kirchberg algebras satisfying suitable freeness conditions [67]. It has recently been shown in [36] that the relation of cocycle conjugacy of automorphisms of the Cuntz algebra $\mathcal{O}_{2}$ is a complete analytic set. This is obtained by analyzing the range of the invariant in a classification result of Izumi from [46] and proving the existence of a Borel inverse for the classifying function. Such a result provides one of the few known lower bounds on the complexity of the classification problem for automorphisms of $\mathrm{C}^{*}$-algebras up to cocycle conjugacy.

\section{The Elliott Classification Program}

3.1. Tensor products. Tensor products play a key role in the theory of $\mathrm{C}^{*}$-algebras. Suppose that $A$ and $B$ are $\mathrm{C}^{*}$-algebras. There is a natural way to define a norm on the algebraic tensor product $A \odot B$ of $A$ and $B$ as complex algebras: concretely represent $A$ and $B$ as closed selfadjoint subalgebras of $B(H)$. Consider the inclusion of $A \odot B$ into $B(H \otimes H)$ given by the action $(a \otimes b)(\xi \otimes \eta)=a \xi \otimes a \eta$ for $a \in A, b \in B$, and $\xi, \eta \in H$. Here $H \bar{\otimes} H$ denotes the Hilbert space tensor product of $H$ by itself, i.e. the completion of the algebraic tensor product $H \odot H$ with respect to the pre-inner product

$$
\left\langle\xi \otimes \eta, \xi^{\prime} \otimes \eta^{\prime}\right\rangle=\left\langle\xi, \xi^{\prime}\right\rangle\left\langle\eta, \eta^{\prime}\right\rangle
$$

The inclusion $A \odot B \subset B(H)$ yields a cross $\mathrm{C}^{*}$-norm on $A \odot B$, i.e. a norm such that $\|a \otimes b\|=$ $\|a\|\|b\|$ and the completion of $A \odot B$ with respect to such a norm is a $\mathrm{C}^{*}$-algebra. In general there may many other cross $\mathrm{C}^{*}$-norms on $A \odot B$. A $\mathrm{C}^{*}$-algebra $A$ for which this does not happen is called nuclear. More precisely a $\mathrm{C}^{*}$-algebra $A$ is nuclear if for any other $\mathrm{C}^{*}$-algebra $B$, there is a unique cross $\mathrm{C}^{*}$-norm on $A \odot B$. The corresponding completion is then simply denoted by $A \otimes B$.

All abelian $\mathrm{C}^{*}$-algebras are nuclear, and in fact the unique tensor product is given by the formula

$$
C(X) \otimes C(Y) \cong C(X \times Y)
$$

where $X \times Y$ denotes the product space. Similarly, full matrix algebras $M_{n}(\mathbb{C})$ are nuclear, and the tensor product $M_{n}(\mathbb{C}) \otimes M_{k}(\mathbb{C})$ can be identified with $M_{n k}(\mathbb{C})$.

The standard example of a $\mathrm{C}^{*}$-algebra that is not nuclear is the reduced $\mathrm{C}^{*}$-algebra $C_{r}^{*}\left(\mathbb{F}_{2}\right)$ of the free group on 2 generators. This is the $\mathrm{C}^{*}$-algebra of operators on the Hilbert space $\ell^{2}\left(\mathbb{F}_{2}\right)$ - with canonical basis $\left(\xi_{h}\right)_{h \in \mathbb{F}_{2}}$ - generated by the unitary operators $u_{g}\left(\xi_{h}\right)=\xi_{g h}$ for $g \in \mathbb{F}_{2}$. In fact the tensor product $C_{r}^{*}\left(\mathbb{F}_{2}\right) \odot C_{r}^{*}\left(\mathbb{F}_{2}\right)$ has the largest possible number of cross 
$\mathrm{C}^{*}$-norms: continuum many [86]. Another important example of $\mathrm{C}^{*}$-algebra that is not nuclear is $B(H)$ when $H$ is the separable infinite-dimensional Hilbert space [85]. Again the amount of cross $\mathrm{C}^{*}$-norms on $B(H) \odot B(H)$ as large as possible: power of the continuum [68].

Nuclearity is a key notion in the theory of $\mathrm{C}^{*}$-algebras. Many equivalent reformulations of nuclearity have been given including Banach-algebraic amenability, the completely positive approximation property, and injectivity or semidiscreteness of the second dual [8, IV.3.1]. In the following we will only consider tensor products of nuclear $\mathrm{C}^{*}$-algebras. A nuclear $\mathrm{C}^{*}$-algebra $A$ is $B$-absorbing if $A \otimes B \cong A$ and self-absorbing if $A \otimes A \cong A$.

The Elliott classification program is an ambitious project aiming at a complete classification of (algebraically) simple nuclear $\mathrm{C}^{*}$-algebras by K-theoretic data [22, 24].

3.2. The Elliott invariant. K-theory was first developed in algebraic geometry by Atiyah and Hirzebruch [3] to study vector bundles by algebraic means (topological K-theory). These ideas were then translated into an algebraic language, leading to algebraic K-theory of Banach algebras and general rings [74]. The K-theoretic machinery was then incorporated in the theory of $\mathrm{C}^{*}$-algebras regarded as noncommutative topology [78].

The K-theory of a $\mathrm{C}^{*}$-algebra $A$ can be described in terms of the $K_{0}$ and $K_{1}$ groups. The ordered $K_{0}$-group $K_{0}(A)$ can be obtained from the Murray-von Neumann semigroup $V(A)$ as the group of formal differences, regarding $V(A)$ as the cone of positive elements inside of $K_{0}(A)$. Such a construction is entirely analogous to the construction of the ordered group $\mathbb{Z}$ from the additive semigroup $\mathbb{N}$. The semigroup $V(A)$ can be described-algebraicallyas the set of equivalence classes of finitely generated projective modules over $A$ up to stable equivalence, where the operation corresponds to the direct sum of modules. Equivalently one can describe $V(A)$ intrinsically in terms of the relation of Murray-von Neumann equivalence of projections. (All projections are assumed to be orthogonal projections onto their range. They can be characterized algebraically as those operators satisfying $p=p^{*}=p^{2}$.) Two projections $p, q \in A$ are equivalent in $A$ if $A$ contains a partial isometry $v$ with support projection $p$ and range projection $q$. This means that $v$ is an isometry from the range of $p$ onto the range of $q$, and identically zero on the orthogonal complement. Equivalently, $v^{*} v=p$ and $v v^{*}=q$. Such a notion captures the fact that $p$ and $q$ have the same dimension relatively to $A$. It was first considered by Murray and von Neumann in their classification theory for von Neumann factors [66]. In order to define $V(A)$ one needs to consider all the matrix amplifications $M_{n}(A)$ and their projections up to equivalence. The semigroup operation correspond to "putting projections on the diagonal", in formulas

$$
p \oplus q=\left[\begin{array}{ll}
p & 0 \\
0 & q
\end{array}\right]
$$

When $A \cong C(X)$ is abelian, projections on matrix amplifications of $A$ correspond to vector bundles over $X$. The notion of Murray-von Neumann equivalence of projections in this case translates into the natural notion of stable equivalence of vector bundles. The $K_{0}$-group thus codes the higher rank topological information on $A$. The $K_{1}$-group $K_{1}(A)$ is defined similarly by considering, instead of projections, unitary elements up to the relation of being connected by a continuous path. More information on K-theory of $\mathrm{C}^{*}$-algebras can be found in [7].

The Elliott invariant of a $\mathrm{C}^{*}$-algebra $A$ comprises the $K_{0}$ and $K_{1}$ groups and the trace simplex $T(A)$ of $A$. A trace on $A$ is a linear functional $\phi$ on $A$ of norm 1 satisfying $\phi(1)=1$ and $\phi(a b)=\phi(b a)$ for $a, b \in A$. This can be seen as a noncommutative analog of a Radon 
probability measure. In fact when $A \cong C(X)$ is abelian, any Radon probability measure $\mu$ on $X$ yields a trace on $C(X)$ given by the integral functional $\int(\cdot) d \mu$, and any trace is of this form. The trace simplex $T(A)$ is a Choquet simplex with respect to the topological and affine structure obtained by regarding of $T(A)$ as a convex subset of the dual $A^{*}$ of $A$.

The last bit of information included in the Elliott invariant is the canonical pairing between $K_{0}$ and traces, obtained by evaluating a trace at (any representative of) an equivalence class of projections. Such a pairing records how the measure-theoretic and the topological structures of the algebra interact. The Elliott invariant is furthermore functorial: a morphism between algebras induces a morphism at the level of invariants.

3.3. AF and $\mathbf{U H F}$ algebras. One of the first instances of classification of a large important class of $\mathrm{C}^{*}$-algebras by $\mathrm{K}$-theoretic invariants is the Elliott-Bratteli classification of approximately finite-dimensional (AF) algebras [10, 20]. For algebras in this class it is shown that any morphisms between the invariants can be lifted to a morphism between the algebras. The main proof technique, which plays an important role in many other further classification results, is an approximate intertwining argument that can be regarded as the $\mathrm{C}^{*}$-algebraic version of Cantor's back-and-forth method.

The uniformly hyperfinite (UHF) algebras are a particularly important class of AF algebras. These are infinite tensor products of algebras of the form $M_{n}(\mathbb{C})$. This class of algebras had been previously classified by Glimm in terms of the corresponding generalized natural number. Such a number has "prime factors" $p^{n}$ where $n \in \mathbb{N} \cup\{\infty\}$ is the supremum of those $k$ such that $M_{p^{k}}(\mathbb{C})$ unitally embeds into the algebra. A UHF algebra is self-absorbing if and only if it is of infinite type, i.e. every prime appears in the associated generalized natural number with infinite exponent.

3.4. Kirchberg algebras. The Elliott-Bratteli classification result for AF algebras was later extended to many other classes of algebras. Most notably a complete classification of purely infinite simple nuclear $\mathrm{C}^{*}$-algebras (Kirchberg algebras) was obtained by Kirchberg and Phillips in [55, 56] modulo the technical assumption that they satisfy the Universal Coefficient Theorem (UCT). It should be mentioned that the UCT holds in all the currently known examples of simple nuclear purely infinite $\mathrm{C}^{*}$-algebras. In fact it is currently a major open problem whether there is any nuclear $\mathrm{C}^{*}$-algebra for which the UCT fails.

The notion of purely infinite $\mathrm{C}^{*}$-algebra is defined in terms of the notion of infinite projection. A projection $p$ in a $\mathrm{C}^{*}$-algebra $A$ is infinite if it is Murray-von Neumann equivalent to a proper subprojection of $p$. This is a projection $q$ distinct from $p$ and dominated by $p$ in the sense that the range of $q$ is contained in the range of $p$ or, equivalently, $p q=q p=q$. Such a definition should be compared with the fact that a set is infinite if and only if it is in bijection with a proper subset (Dedekind-infiniteness). A simple $\mathrm{C}^{*}$-algebra is then purely infinite if it has an abundance of infinite projections: for every nonzero element $x$ of $A$ the subalgebra $x^{*} A x$ contains an infinite projection. A Kirchberg algebra is a simple nuclear purely infinite $\mathrm{C}^{*}$-algebra. Kirchberg algebras do not have any trace. Moreover the order structure on the $K_{0}$-group trivializes, and every element is positive. Therefore the Elliott invariant of a Kirchberg algebra $A$ reduces to the pair of (countable, discrete) abelian groups $K_{0}(A)$ and $K_{1}(A)$.

Fundamental examples of purely infinite simple nuclear $\mathrm{C}^{*}$-algebras are the Cuntz algebras $\mathcal{O}_{2}$ and $\mathcal{O}_{\infty}$. The Cuntz algebra $\mathcal{O}_{2}$ is the $\mathrm{C}^{*}$-algebra generated by two isometries $v_{1}, v_{2}$ with complementary ranges. This means that $v_{1}, v_{2} \in B(H)$ are linear isometries with images two 
complementary subspaces of $H$ or, equivalently, $v_{1} v_{1}^{*}+v_{2} v_{2}^{*}=v_{1}^{*} v_{1}=v_{2}^{*} v_{2}=1$. The Cuntz algebra $\mathcal{O}_{\infty}$ is generated by infinitely many isometries $\left(v_{n}\right)_{n \in \mathbb{N}}$ with mutually orthogonal ranges, i.e. satisfying $v_{i}^{*} v_{i}=1$ and $v_{i}^{*} v_{j}=0$ for $i \neq j$. One can similarly define the Cuntz algebra $\mathcal{O}_{n}$ for any $n \geq 2$. Such algebras were first defined and studied by Cuntz in [14]. Cuntz showed that the isomorphism class of these algebras does not depend on the choice of the generating isometries. Moreover they are simple and purely infinite. Their K-theory was computed in [15]: they all have trivial $K_{1}$-group, while $K_{0}\left(\mathcal{O}_{\infty}\right) \cong \mathbb{Z}$ and $K_{0}\left(\mathcal{O}_{2}\right)$ is trivial. More generally $K_{0}\left(\mathcal{O}_{n}\right) \cong \mathbb{Z} /(n-1) \mathbb{Z}$. The first step in the Kirchberg-Phillips classification consists in showing that, if $B$ is a simple nuclear $\mathrm{C}^{*}$-algebra, then $\mathcal{O}_{2}$ is $B$-absorbing, and $B$ is purely infinite if and only if it is $\mathcal{O}_{\infty}$-absorbing.

3.5. The Jiang-Su algebra. The possible extent of the Elliott classification program has been recently limited by breakthroughs due Rørdam and Toms. In [73, 81, 82] they showed that in general the Elliott invariant is not a complete invariant for simple nuclear $\mathrm{C}^{*}$-algebras. In fact continuum many pairwise nonisomorphic simple nuclear $\mathrm{C}^{*}$-algebras with the same Elliott invariant are constructed in [83]. These algebras are distinguished by another invariant, the radius of comparison, which is a noncommutative analog of the mean dimension of dynamical systems [58, 59]. The radius of comparison is detected by the first order theory of the $\mathrm{C}^{*}$ algebra seen as a structure in continuous logic [5, 28]. It therefore remains open the - unlikelypossibility that the Elliott invariant together with the first order theory provides a complete invariant for simple nuclear $\mathrm{C}^{*}$-algebras; see [27].

Many efforts have been recently dedicated to isolate the class of "well behaved" C*-algebras for which the Elliott classification program can be successfully recasted. In these efforts the Jiang-Su algebra $\mathcal{Z}$ has a role of paramount importance. This is an infinite-dimensional simple nuclear $\mathrm{C}^{*}$-algebra that is a tensorial "neutral element" at the level of invariants. It was first constructed by Jiang and $\mathrm{Su}$ in [48] as limit of dimension drop algebras. These are algebras of the form

$$
\left\{f \in C\left([0,1], M_{p}(\mathbb{C}) \otimes M_{q}(\mathbb{C})\right): f(0) \in M_{p}(\mathbb{C}) \otimes 1, f(1) \in 1 \otimes M_{q}(\mathbb{C})\right\}
$$

endowed with the canonical trace coming from the Lebesgue measure on [0,1]. The Jiang-Su construction was shown to be of Fraïssé-theoretical nature in [18].

If $A$ is any simple nuclear $\mathrm{C}^{*}$-algebra, then $A \otimes \mathcal{Z}$ and $A$ have the same Elliott invariant. It follows that it is a necessary condition for $A$ to be classifiable that $A$ be $\mathcal{Z}$-absorbing. The notion of $\mathcal{Z}$-absorption is the subject of an important conjecture due to Toms and Winter. Such a conjecture asserts that, for simple nuclear $\mathrm{C}^{*}$-algebras, $\mathcal{Z}$-absorption is equivalent to other two regularity properties of topological and cohomological nature, respectively: finite nuclear dimension and strict comparison. Such a conjecture has been by now verified in a vast number of cases including all $\mathrm{C}^{*}$-algebras whose trace simplex is "small"; see [9] and references therein.

3.6. Strongly self-absorbing $\mathbf{C}^{*}$-algebras. The Jiang-Su algebra $\mathcal{Z}$, the Cuntz algebras $\mathcal{O}_{2}$ and $\mathcal{O}_{\infty}$, and the infinite-type UHF algebras all play a key role in the classification program of $\mathrm{C}^{*}$-algebras. The fundamental properties that make these algebras stand out have been isolated in [84]. A (necessarily nuclear) $\mathrm{C}^{*}$-algebra is strongly self-absorbing if the embedding $a \mapsto a \otimes 1$ of $A$ into $A \otimes A$ is approximately unitarily equivalent to an isomorphism. This means that there exist an isomorphism $\gamma: A \rightarrow A \otimes A$ and a sequence $\left(u_{n}\right)$ of unitaries of $A$ such that $\left\|\gamma(a)-u_{n}(a \otimes 1) u_{n}^{*}\right\| \rightarrow 0$. A strongly self-absorbing $\mathrm{C}^{*}$-algebra $A$ is in particular 
self-absorbing, and in fact isomorphic to the infinite tensor product $A^{\otimes \mathbb{N}}$ of copies of $A$. The algebras $\mathcal{Z}, \mathcal{O}_{2}, \mathcal{O}_{\infty}$, the infinite type UHF algebras and their tensor products are the only currently known examples of strongly self-absorbing $\mathrm{C}^{*}$-algebras.

3.7. Borel complexity of $\mathbf{C}^{*}$-algebras. The analysis of the classification problem of $\mathrm{C}^{*}$ algebras from the perspective of Borel complexity theory has been initiated in [30]. The Elliott invariant has been shown to be Borel-computable with respect to any natural parametrization of $\mathrm{C}^{*}$-algebras [29]. As observed in [26, §3], by combining results from [23, 35, 64, 76, 89] one can conclude that the following relations all have maximum complexity among equivalence relations that are classifiable by the orbits of a Polish group action:

- Isomorphism of (Elliott-classifiable) $\mathrm{C}^{*}$-algebras;

- Homeomorphism of compact metrizable spaces;

- Affine homeomorphism of Choquet simplices;

- Isometry of Banach spaces;

- Isometry of complete separable metric spaces.

While some of the classification problems above are perceived to be less intractable than others, they have in fact the same complexity from the perspective of invariant descriptive set theory. However the functorial nature of the classification problem for $\mathrm{C}^{*}$-algebras is not taken into account by this analysis. Functorial Borel complexity is a refinement introduced in [61] of the usual notion of Borel complexity, aiming at capturing the complexity of classifying the objects of a category in an explicit and functorial way. It is conceivable that some of the classes above might have distinct functorial complexity.

\section{Conjugacy}

In this section we will consider the relation of conjugacy for automorphisms of a $\mathrm{C}^{*}$-algebra $A$. When $A$ is abelian, $A \cong C(K)$ for some compact metrizable space, and Aut $(A) \cong$ Homeo $(K)$. Therefore the relation of conjugacy for automorphisms of $A$ coincides with the relation of conjugacy of homeomorphisms of $K$. The latter equivalence relation has been studied for particular instances of $K$. For example it has been shown in [42, $\S 4.2]$ that the homeomorphisms of the unit interval $[0,1]$ are classifiable by countable structures. However an increase of dimension entails an increase of complexity: the homeomorphisms of the unit square $[0,1]^{2}$ are not classifiable by countable structures [42, 4.3]. This result was one of the first applications of Hjorth's turbulence theory, developed therein.

When $K$ is zero-dimensional, $K$ is the Stone space of a countable Boolean algebra $\mathcal{B}$, and $\operatorname{Homeo}(K) \cong \operatorname{Aut}(\mathcal{B})$. Therefore conjugacy of homeomorphisms of $K$ coincides with conjugacy of automorphisms of $\mathcal{B}$. Since Aut $(\mathcal{B})$ is a closed subgroup of $S_{\infty}$, it follows that the latter equivalence relation is classifiable by countable structures. When $\mathcal{B}$ is the countable atomless Boolean algebra, its Stone space $K$ is the Cantor set. The relation of conjugacy of homeomorphisms of $K$ has been shown to have maximum complexity among relations that are classifiable by countable structures in [12].

Some positive classification results up to conjugacy for automorphisms of finite order by K-theoretic invariants have been obtained by Izumi [46, 47]. These results assume the automorphisms (or their "dual actions") to have suitable freeness conditions such as the Rokhlin property 71. Moreover they apply to classes of algebras that are classifiable in the sense of the Elliott program, such as the class of Kirchberg algebras; see 33.4 . The starting point of Izumi 
analysis is the Kirchberg-Phillips classification result, and the study of automorphisms at the level of the invariants.

In the rest of this section we will present the following nonclassifiability result for automorphisms of $\mathcal{Z}$-absorbing $\mathrm{C}^{*}$-algebras.

Theorem 4.1 (Kerr-Lupini-Phillips, 2014). Suppose that $A$ is a $\mathrm{C}^{*}$-algebra such that $A \otimes \mathcal{Z} \cong$ $A$. Then the automorphisms of $A$ are not classifiable up to conjugacy by countable structures.

Theorem 4.1 in particular rules out classification by K-theoretic data in view of the Borelcomputability of K-theory [29]. As observed in 33.5 , the class of $\mathcal{Z}$-absorbing $\mathrm{C}^{*}$-algebras contains all $\mathrm{C}^{*}$-algebras that fall under the scope of the classification program. The proof strategy for Theorem 4.1 is to establish generic turbulence of the conjugation action $\operatorname{Aut}(\mathcal{Z}) \curvearrowright \operatorname{Aut}(\mathcal{Z})$, and then use Hjorth's turbulence theorem together with the canonical embedding $\operatorname{Aut}(\mathcal{Z}) \hookrightarrow$ $\operatorname{Aut}(A \otimes \mathcal{Z}) \cong \operatorname{Aut}(A)$ to deduce nonclassifiability in $\operatorname{Aut}(A)$.

Recall from 33.6 that the Jiang-Su algebra is a particular instance of an important class of algebras known as strongly self-absorbing [84, Definition 1.1]. Such a property in particular implies that $\mathcal{Z}$ is isomorphic to the infinite tensor product $\mathcal{Z}^{\otimes \mathbb{Z}}$ of copies of $\mathcal{Z}$. As a consequence after replacing $\mathcal{Z}$ with $\mathcal{Z}^{\otimes \mathbb{Z}}$ one can consider the shift automorphism $\sigma$ of $\mathcal{Z}^{\otimes \mathbb{N}}$ associated with the shift $n \mapsto n-1$ on $\mathbb{Z}$. A key property of the shift $\sigma$ is being malleable [54, Lemma 2.6]. This means that there is a continuous path $\left(\rho_{t}\right)_{t \in[0,1]}$ in Aut $\left(\mathcal{Z}^{\otimes \mathbb{Z}} \otimes \mathcal{Z}^{\otimes \mathbb{Z}}\right)$ such that $\rho_{0}$ is the identity, $\rho_{1}$ is the flip $x \otimes y \mapsto y \otimes x$, and $\rho_{t} \circ(\sigma \otimes \sigma)=(\sigma \otimes \sigma) \circ \rho_{t}$ for every $t \in[0,1][54$, Definition 2.5]. The notion of malleability plays a key role in Popa's deformation-rigidity theory for von Neumann algebras and $\mathrm{II}_{1}$ factors [72]. Such a notion turns out to be of fundamental importance also in this $\mathrm{C}^{*}$-algebraic setting. In particular it is the key property that allows one to establish the following result.

Proposition 4.2. The the shift automorphism $\sigma$ of $\mathcal{Z}^{\otimes \mathbb{Z}} \cong \mathcal{Z}$ is a turbulent point with dense orbit for the conjugation action $\operatorname{Aut}(\mathcal{Z}) \curvearrowright \operatorname{Aut}(\mathcal{Z})$.

Proposition 4.2 is obtained via a factor exchange argument applied to the tensor product $\mathcal{Z}^{\otimes \mathbb{Z}} \otimes \mathcal{Z}^{\otimes \mathbb{Z}}$. Malleability of the shift is used to carry out the exchange via a continuous path of unitaries in a way that commutes with the shift itself. Density of the shift is a consequence of work of Sato on stability properties of the shift and, more generally, automorphisms of $\mathcal{Z}$ satisfying a suitable freeness condition [77].

In order to conclude the proof that the conjugation action $\operatorname{Aut}(\mathcal{Z}) \curvearrowright \operatorname{Aut}(\mathcal{Z})$ is generically turbulent, one then needs show that every orbit is meager. This can be established as an application of a result of Rosendal that infers meagerness of conjugacy classes from a density condition for periodic elements.

Lemma 4.3 (Rosendal). Suppose that $G$ is a Polish group. If for every infinite subset $I$ of $\mathbb{N}$ the set of $g \in G$ such that $g^{n}=1$ for some $n \in I$ is dense, then $G$ has meager conjugacy classes.

The hypothesis of Lemma 4.3 imply the existence of a collection of conjugation-invariant comeager subsets of $G$ with trivial intersection. This directly imply meagerness of conjugacy classes; see [75, Proposition 18] and [51, page 9].

In order to conclude that Aut $(\mathcal{Z})$ satisfies the hypothesis of Lemma 4.3, one can observe that the shift $\sigma$ can be approximated by "periodic shifts" of sufficiently large period. Formally these 
are the automorphisms of $\mathcal{Z}^{\otimes \mathbb{N}} \cong \mathcal{Z}$ associated with cyclic permutations of $\mathbb{Z}$

$$
(-n-n+1-n+2 \cdots m-1 m)
$$

for $n, m \in \mathbb{N}$. This suffices in view of the fact that the shift has dense conjugacy class.

We now explain how to deduce Theorem 4.1 from Proposition 4.2, Suppose that $A$ is a $\mathcal{Z}$ absorbing $\mathrm{C}^{*}$-algebra. Identifying $A$ with $A \otimes \mathcal{Z}$ we can regard $\operatorname{Aut}(\mathcal{Z})$ as a closed subgroup of Aut $(A \otimes \mathcal{Z})$ via the inclusion $\gamma \mapsto i d_{A} \otimes \gamma$. One can then argue by contradiction that, if the automorphisms of $A \otimes \mathcal{Z}$ are classifiable by countable structures, then the relation for elements of Aut $(\mathcal{Z})$ of being conjugate inside Aut $(A \otimes \mathcal{Z})$ must have a comeager class. However the latter fact can be excluded essentially via the same argument that shows that $\operatorname{Aut}(\mathcal{Z})$ has meager conjugacy classes.

\section{UNITARY EQUIVALENCE}

In this section we consider the relation of unitary equivalence of automorphisms of $\mathrm{C}^{*}$ algebras. Suppose that $A$ is a $\mathrm{C}^{*}$-algebra. Recall that any unitary element $u$ of $A$ induces an automorphism $\operatorname{Ad}(u)$ of $A$ defined by $x \mapsto u x u^{*}$. Automorphisms of this form are called inner and form a normal subgroup $\operatorname{Inn}(A)$ of Aut $(A)$. Two automorphisms of $A$ are unitarily equivalent if they belong to the same coset of $\operatorname{Inn}(A)$. It should be noted that $\operatorname{Inn}(A)$ is the continuous homomorphic image of the unitary group $U(A)$ of $A$ under the map $u \mapsto \operatorname{Ad}(u)$. Therefore, being the continuous homomorphic image of a Polish group, it is a Borel subset of Aut $(A)$ [49, Exercise 15.15]. Moreover a standard argument in descriptive set theory allows one to conclude that the relation of unitary equivalence of automorphisms of $A$ is smooth if and only if $\operatorname{Inn}(A)$ is a closed subgroup of $\operatorname{Aut}(A)$. In fact if $\operatorname{Inn}(A)$ is closed then the quotient group Aut $(A) / \operatorname{Inn}(A)$ is Polish, and its elements parametrize the cosets of $\operatorname{Inn}(A)$. Conversely if unitary equivalence is smooth then by turning Borel sets into clopen sets [49, §13.A] one can find a finer topology on Aut $(A)$ that makes such Inn $(A)$ closed. By the uniqueness of the Polish topology in a Polish group, such a topology coincides with the original one, and hence Inn $(A)$ was already closed.

An explicit classification of automorphisms of $A$ up to unitary equivalence has been obtained in [70] when $A$ has continuous trace. In particular this classification shows that in the continuous trace case such a relation is smooth. Conversely it has been shown in [69] that if $A$ does not have continuous trace, then the relation of unitary equivalence of automorphisms is not smooth. The last result has been strengthened in [62] by showing that when $A$ does not have continuous trace, then in fact the automorphisms of $A$ are not classifiable up to unitary equivalence by countable structures. In this section we will present an overview of these results, after introducing the notion of continuous trace $\mathrm{C}^{*}$-algebra.

A central sequence in a $\mathrm{C}^{*}$-algebra $A$ is a bounded sequence $\left(a_{n}\right)$ in $A$ such that the commutators $a_{n} b-b a_{n}$ converge to 0 for any $b \in A$. A central sequence is trivial if there is a sequence $\left(z_{n}\right)$ in the center of $A$ such that $\left\|a_{n}-z_{n}\right\| \rightarrow 0$ when $n \rightarrow+\infty$. (The center $Z(A)$ of $A$ is the subalgebra of those elements of $A$ that commute with any other element of $A$.) The $\mathrm{C}^{*}$-algebra $A$ has continuous trace if every central sequence in $A$ is trivial. While this is not the original definition of continuous trace $\mathrm{C}^{*}$-algebras, it has been proved to be equivalent by Akemann and Pedersen [1] building on previous work of Elliott [21].

The fact that $\operatorname{Inn}(A)$ is closed in Aut $(A)$ if and only if $A$ has continuous trace observed in [21, 69] - follows from a standard fact in the theory of $\mathrm{C}^{*}$-algebras: a $\mathrm{C}^{*}$-algebra is generated by 
its unitary elements $[8$, II.3.2.16]. In particular the center of its unitary group coincides with the intersection of $U(A)$ with the center of $A$. Recall also the classical principles from descriptive set theory that a Borel bijection between standard Borel spaces is a Borel isomorphism [49, Corollary 15.2], and a Borel homomorphism between Polish groups is continuous [49, Exercise 9.16].

If $\operatorname{Inn}(A)$ is closed in $\operatorname{Aut}(A)$, then the map $w \mapsto \operatorname{Ad}(w)$ from the unitary group $U(A)$ of $A$ to $\operatorname{Inn}(A)$ induces, passing to the quotient, a Polish group isomorphism from the quotient of $U(A)$ by its center to $\operatorname{Inn}(A)$. If $\left(w_{n}\right)$ is a central sequence in $A$, then $\operatorname{Ad}\left(w_{n}\right)$ converges to the identity automorphism of $A$. Henceforth $\left(w_{n}\right)$ converges to the identity in the quotient of $U(A)$ by its center. This means that there is a sequence $\left(z_{n}\right)$ in $Z(A) \cap U(A)$ such that $\left\|u_{n}-z_{n}\right\| \rightarrow 0$. In other words $\left(w_{n}\right)$ is a trivial central sequence. The converse implication can be proved just reversing the argument above.

Such a result admits the following strengthening, which can be seen as an instance of the principle that "when it is bad, it is worse"; see [62, Theorem 1.1].

Theorem 5.1. If $A$ is a $C^{*}$-algebra that does not have continuous trace, then the automorphisms of $A$ are not classifiable up to unitary equivalence by countable structures.

Theorem 5.1 in particular implies a dichotomy for the complexity of the relation of unitary equivalence of automorphisms of $\mathrm{C}^{*}$-algebras. Such a relation is either smooth, or not classifiable by countable structures. The same phenomenon has been observed for the relation of unitary equivalence of irreducible representations of locally compact groups and $\mathrm{C}^{*}$-algebras [25, 41, 53].

In the rest of this section we will present the main ideas in the proof of Theorem [5.1. Again Hjorth's theory of turbulence will play a central role. One of the first examples of turbulent action provided by Hjorth is the action of $\ell^{1}$ on $\mathbb{R}^{\mathbb{N}}$ by translation [42, $\left.\S 3.3\right]$. Here $\ell^{1}$ is regarded as an (additive) Polish group endowed with the topology induced by the $\ell^{1}$-norm, while $\mathbb{R}^{\mathbb{N}}$ is endowed with the product topology.

Hjorth's turbulence theorem together with turbulence of the translation action of $\ell^{1}$ on $\mathbb{R}^{\mathbb{N}}$ yields the following nonclassifiability criterion; see [62, Criterion 3.3].

Criterion 5.2. Suppose that $E$ is an equivalence relation on a standard Borel space $X$. If there is a Borel function $f:(0,1)^{\mathbb{N}} \rightarrow X$ such that

(1) $f(\mathbf{x}) E f\left(\mathbf{x}^{\prime}\right)$ whenever $\mathbf{x}, \mathbf{x}^{\prime} \in(0,1)^{\mathbb{N}}$ are such that $\mathbf{x}-\mathbf{x}^{\prime} \in \ell^{1}$, and

(2) the preimage under $f$ of any $E$-class is meager,

then $E$ is not classifiable by countable structures.

Criterion 5.2 will be the main tool to establish Theorem 5.1 . Let $A$ be a $\mathrm{C}^{*}$-algebra that does not have continuous trace. Thus $A$ contains a nontrivial central sequence $\left(x_{n}\right)$. We will assume for simplicity that $\left(x_{n}\right)$ is nontrivial in the following stronger sense: there is a central sequence $\left(y_{n}\right)$ in $A$ such that the commutators $x_{n} y_{n}-y_{n} x_{n}$ do not converge to 0 . This covers most interesting cases, including when the $\mathrm{C}^{*}$-algebra $A$ is simple.

After replacing $x_{n}$ with $x_{n}^{*} x_{n}$ we can assume that the $x_{n}$ 's are positive. This means that they are positive linear operators in any concrete representation of $A$ as a subalgebra of $B(H)$. The idea is now to use the sequence $\left(x_{n}\right)$ to produce, with an eye at Criterion 5.2. "many" automorphisms of $A$ indexed by sequences $\left(t_{n}\right)$ in $(0,1)^{\mathbb{N}}$. In order to define these automorphisms we need to turn the $x_{n}$ 's into unitary elements. This can be achieved by taking exponentials. 
Recall that if $x$ is a positive real number then $\exp (i x)=\sum_{k \in \mathbb{N}} \frac{1}{k !}(i x)^{k}$ is a complex number of modulus 1, i.e. a unitary element of $\mathbb{C}$. The same fact-with similar proof - applies when $\mathbb{C}$ is replaced by an arbitrary $\mathrm{C}^{*}$-algebra. If $x$ is a positive element of $A$, then $\exp (i x)=$ $\sum_{k \in \mathbb{N}} \frac{1}{k !}\left(i x^{k}\right)$ is a well defined unitary element of $A$.

We can then define for $\left(t_{n}\right) \in(0,1)^{\mathbb{N}}$ an automorphism $\alpha_{\left(t_{n}\right)}$ of $A$ as the limit of the sequence of inner automorphisms $\operatorname{Ad}\left(\exp \left(i t_{n} x_{n}\right)\right)$ associated with the unitary elements $\exp \left(i t_{n} x_{n}\right)$. The fact that $\left(x_{n}\right)$ is a central sequence guarantees, perhaps after passing to a subsequence, that such automorphisms are well defined. The map $f: \mathbf{t} \mapsto \alpha_{\mathbf{t}}$ is easily seen to be Borel (in fact, continuous). Moreover if $\mathbf{t}, \mathbf{s} \in(0,1)^{\mathbb{N}}$ are such that $\mathbf{t}-\mathbf{s} \in \ell^{1}$ then $\alpha_{\mathbf{t}}=\operatorname{Ad}(u) \circ \alpha_{\mathbf{s}}$ where $u$ is the limit inside the unitary group of $A$ of the sequence

$$
\left(\exp \left(i t_{1} x_{1}\right) \cdots \exp \left(i t_{n} x_{n}\right) \exp \left(-i s_{n} x_{n}\right) \cdots \exp \left(-i s_{1} x_{1}\right)\right)_{n \in \mathbb{N}} .
$$

Thus $f$ satisfies Condition (1) of Criterion 5.2, In order to establish Condition (2), one has to show that the preimage under $f$ of any unitary equivalence class is meager. If $X \subset(0,1)^{\mathbb{N}}$ is nonmeager, then it is easy to see that there are $\mathbf{t}, \mathbf{s} \in X$ such that $\left|t_{n}-s_{n}\right|>\frac{1}{2}$ for infinitely many $n \in \mathbb{N}$. One can then use the sequence $\left(y_{n}\right)$ to argue that, for such a choice of $\mathbf{s}$ and $\mathbf{t}$ in $X, \alpha_{\mathbf{s}} \circ \alpha_{\mathbf{t}}^{-1}$ is not inner. (More details can be found in [62, Proposition 5.6].) Therefore $\alpha_{\mathbf{s}}$ and $\alpha_{\mathbf{t}}$ are not unitarily equivalent. This concludes the proof that the preimage under $f$ of any unitary equivalence class is meager, and $f$ satisfies the assumptions of Criterion 5.2 .

\section{Cocycle conjugacy}

Recall that two automorphisms $\alpha$ and $\beta$ are cocycle conjugate if a conjugate of $\alpha$ is unitarily equivalent to $\beta$. In formulas $\alpha=\operatorname{Ad}(u) \circ \gamma \circ \beta \circ \gamma^{-1}$ for some unitary element $u$ of $A$ and some automorphism $\gamma$ of $A$. The majority of positive classification results for automorphisms of $\mathrm{C}^{*}$-algebras are up to cocycle conjugacy. Yet, not much is known in general, and most of the results hold under the additional assumption that the automorphisms have the (weak) Rokhlin property. Such a property prescribes the existence of a noncommutative analog of the Rokhlin tower from ergodic theory. It turns out that under very general assumptions automorphisms with the Rokhlin property exist, and in fact form a dense $G_{\delta}$ set [1]. Little is known about the complexity of the relation of cocycle conjugacy outside the (comeager) set of automorphism with the Rokhlin property.

Kishimoto has shown in [57] that for UHF algebras any two automorphisms with the Rokhlin property are cocycle conjugate. A similar result has been obtained by Sato for automorphisms of the Jiang-Su algebra [77]. In both cases the proof relies on the characterization of automorphisms with the Rokhlin property as automorphisms all of whose powers are outer in a strong sense. This indicates that the Rokhlin property can be seen as a quantized analog of freeness for topological dynamical systems. The other fundamental feature of automorphism with the Rokhlin property that is used in Kishimoto and Sato's result is stability. Stability guarantees that if $\alpha$ is an automorphism with the Rokhlin property and $u$ is a unitary, then $u$ can be approximated with a unitary of the form $v \alpha\left(v^{*}\right)$ for some unitary $v$. This allows one two perturb any two automorphisms with the Rokhlin property to be cocycle conjugate.

In the case of Kirchberg algebras satisfying the Universal Coefficient Theorem, the KirchbergPhillips classification mentioned in $\$ 3.4$ also provides information about automorphisms. Recall that such a classification result asserts that UCT Kirchberg algebras are classified by their Elliott 
invariants. Since Kirchberg algebras have no traces, the Elliott invariant reduces to the $K_{0}$ and $K_{1}$ groups. A key ingredient of the Kirchberg-Phillips classification result consists in showing that two morphisms between Kirchberg algebras induces the same morphism at the level of invariants if and only if they are asymptotically unitarily equivalent. Such a notion is the analog of unitary equivalence where one replaces a single unitary with a continuous path. It was later shown by Nakamura that, under the additional assumption of the Rokhlin property, asymptotic unitary equivalence implies cocycle conjugacy [67, Theorem 5]. Again the stability of Rokhlin property automorphisms plays a major role. Combining Nakamura's result and the KirchbergPhillips classification, one obtains that Rokhlin property automorphisms of UCT Kirchberg algebras are cocycle conjugate if and only if the corresponding morphisms at the level of the Elliott invariant are conjugate [67, Theorem 9].

In the particular case of the Cuntz algebra $\mathcal{O}_{2}$, both the $K_{0}$ and the $K_{1}$ groups are trivial. Therefore it follows from Nakamura's classification that any two automorphisms of $\mathcal{O}_{2}$ with the Rokhlin property are cocycle conjugate. Beyond the class of automorphisms with the Rokhlin property, a classification result has been obtained by Izumi for strongly approximately inner symmetries of $\mathcal{O}_{2}$ [46, 47]. Again the Kirchberg-Phillips classification of UCT Kirchberg algebras plays a key role, together with Kirchberg's absorption theorem asserting that $\mathcal{O}_{2} \otimes B \cong \mathcal{O}_{2}$ for any simple nuclear $\mathrm{C}^{*}$-algebra $B$.

A symmetry is an automorphism of order 2. A symmetry is strongly approximately inner if it is approximated by inner automorphisms induced by unitaries that are approximately of order 2 and are approximately fixed by the given automorphism. It should be mentioned that no symmetry of $\mathcal{O}_{2}$ that is not strongly approximately inner is currently known. In fact very recent results suggest that the problem of the existence of such symmetries is tightly connected with the problem of the existence of nuclear $\mathrm{C}^{*}$-algebras for which the UCT fails.

The invariant involved in Izumi's classification is the crossed product, which is a $\mathrm{C}^{*}$-algebra encoding the action. The crossed product is one of the most important constructions in $\mathrm{C}^{*}$ dynamics [8, §II.10]. Succinctly, if $A$ is a $\mathrm{C}^{*}$-algebra and $\alpha$ is an automorphism, then the corresponding crossed product $A \rtimes_{\alpha} \mathbb{Z}$ is the "universal" $\mathrm{C}^{*}$-algebra generated by $A$ and a unitary $u$ that "implements $\alpha$ " in the sense that $u x u^{*}=\alpha(x)$ for every $x \in A$.

It follows from the Izumi and Kirchberg-Phillips classification results that, under the additional assumption that the crossed product satisfies the UCT, the K-theory of the crossed product provides a complete invariant up to cocycle conjugacy for strongly approximately inner symmetries of $\mathcal{O}_{2}$. The range of the invariants has also been computed by Izumi. This is the collection of all pairs of countable uniquely 2-divisible abelian groups. (An abelian group is 2divisible if every element can be written as double of some element. When such a representation is unique, the group is uniquely 2-divisible.) The fact that such a classifying map is Borel can be seen using the methods from [29]. It has been shown in [36] that such a map admits a Borel inverse. For simplicity the attention is restricted there to algebras with trivial $K_{1}$-group.

Proposition 6.1 (Gardella-Lupini, 2014). There is a Borel map $G \mapsto \alpha_{G}$ from the space of uniquely 2-divisible countable abelian group to the space of strongly approximately inner symmetries of $\mathcal{O}_{2}$ such that $G$ is the $K_{0}$-group of the crossed product of $\mathcal{O}_{2}$ by $\alpha_{G}$, and the $K_{1}$-group of such a crossed product is trivial.

Such a result together with Izumi's classification shows the following: 
Theorem 6.2 (Gardella-Lupini, 2014). The relation of isomorphism of uniquely 2-divisible countable abelian groups is Borel reducible to the relation of cocycle conjugacy of (strongly approximately inner) symmetries of $\mathcal{O}_{2}$.

A minor modification of the argument Downey-Montalbán from [17] — see also [44] - shows that the relation of isomorphism of torsion free 2-divisible countable abelian group is a complete analytic set.

Recall that if $A, B$ are analytic subsets of standard Borel spaces $X, Y$, then $A$ is Wadgereducible to $B$, in formulas $A \leq_{W} B$, if there is a Borel function $f: X \rightarrow Y$ such that $A$ is the preimage of $B$ under $f$. An analytic set is complete if it is a $\leq_{W}$-maximum element of the class of analytic sets. The well known fact that there are analytic sets that are not Borel shows in particular that a complete analytic set is not Borel.

The canonical example of a complete analytic set is the set of ill-founded trees on $\mathbb{N}$ inside the space of all trees. (A tree is ill-founded if it has an infinite branch, and well-founded otherwise.) In order to show that the relation of isomorphism of 2-divisible torsion-free abelian groups is a complete analytic set, it is enough to find a Wadge reduction from the set of ill-founded trees to such a relation. To facilitate the task one can use the following theorem: there are Borel maps $R, S$ from trees to trees such that, for any tree $T$

- if $T$ is ill-founded, then $R(T) \cong S(T)$ is ill-founded, and

- if $T$ is well-founded, then $R(T)$ is well-founded and $S(T)$ is ill-founded.

In fact it suffices to define maps $R, S$ as above from trees to countable linear orders. One can then just take the tree of finite descending chains of the linear order. The existence of such maps has been observed in [17, Lemma 3.1]. The key idea is to use Harrison's theorem from [40], asserting that a pseudo-well order $L$, i.e. a countable linear with no infinite descending chains that are hyperarithmetic in $L$, is ill-founded if and only if it is of the form $\omega_{1}^{L}(1+\mathbb{Q})+\alpha$ for some $\alpha<\omega_{1}^{L}$. To define $R(T)$, first pair up $T$ with the complete binary tree. This gives a tree $T_{1}$ with either none or uncountably many infinite branches. Then express the set of branches of $T_{1}$ that are not hyperarithmetic in $T$ as $\left\{x \in \omega^{<\omega}: \widehat{T}(x)\right.$ is ill-founded $\}$ for some tree $\widehat{T}$ on $\omega \times \omega$ computable in $T$. (Here $\widehat{T}(x)$ denotes the section tree of $\widehat{T}$ corresponding to $x$.) Let now $L$ be the Brower-Kleene order of $\widehat{T}$, and $R(T)=L+L+L+\cdots=L \omega$. Observe that if $T$ is well-founded, then $R(T)$ is well-founded, otherwise $R(T) \cong \omega_{1}^{T}(1+\mathbb{Q})$ by Harrison's theorem. Similarly one can define a Borel-computable map such that $S(T) \cong \omega_{1}^{T}(1+\mathbb{Q})$ for every $T$.

In view of such a result, to conclude the proof that the isomorphism relation of countable 2-divisible torsion-free abelian groups is a complete analytic set, it is enough to assign in a Borel fashion to any tree $T$ a countable 2-divisible torsion free abelian group $T_{G}$ in such a way that

- if $T \cong T^{\prime}$ then $G_{T} \cong G_{T^{\prime}}$, and

- if $G_{T} \cong G_{T^{\prime}}$ then exactly one between $T$ and $T^{\prime}$ is well-founded.

To perform such a construction, fix enumerations $\left(p_{n}\right)$ and $\left(q_{n}\right)$ of pairwise disjoint sets of odd primes. Suppose that $T$ is a tree, with vertex set $V$. Let $\mathbb{Q}^{(V)}$ be the group of formal sums $\sum_{v \in V} q_{v} v$ where $q_{v} \in \mathbb{Q}$ and all but finitely many $q_{v}$ 's are zero. Define $G_{T}$ to be the subgroup of $\mathbb{Q}^{(V)}$ spanned by elements of the form $\frac{1}{2 p_{n}^{k}} v$ and $\frac{1}{2 q_{n}^{k}}(v+w)$ where $k \in \mathbb{N}, v$ is a vertex of $T$ of height $n$, and $w$ is an immediate successor of $v$ in $T$. 
It is clear from the construction that isomorphic trees yield isomorphic groups. In order to verify the second condition above, it is enough to argue that one can detect the existence of an infinite branch of $T$ by looking at group-theoretic properties of $G_{T}$. In fact one can verify that infinite branches in $T$ correspond to sequences $\left(g_{n}\right)$ in $G_{T}$ such that $g_{n}$ is infinitely $p_{n}$-divisible, and $g_{n}+g_{n+1}$ is infinitely $q_{n}$-divisible. This concludes the proof that the relation of isomorphism of 2-divisible torsion free abelian group is a complete analytic set. From this and Theorem 6.2 one can deduce the following corollary.

Corollary 6.3. The relation of cocycle conjugacy of automorphisms of $\mathcal{O}_{2}$ is a complete analytic set.

In fact the same holds for the relation of conjugacy of automorphisms of $\mathcal{O}_{2}$, essentially by the same argument. Moreover one can replace symmetries with automorphisms of order $p$, and 2-divisible groups with $p$-divisible groups for any prime $p$ using a construction of Barlak and Szabó [4]. Not much else is known in general about cocycle conjugacy of automorphisms of $\mathcal{O}_{2}$.

Problem 6.4. Is the relation of cocycle conjugacy of automorphisms of $\mathcal{O}_{2}$ classifiable by countable structures?

In fact in Problem 6.4 one can replace $\mathcal{O}_{2}$ with any other simple nuclear $\mathrm{C}^{*}$-algebra; see [36].

\section{REFERENCES}

[1] Charles A. Akemann and Gert K. Pedersen, Central sequences and inner derivations of separable $C^{*}$-algebras, American Journal of Mathematics 101 (1979), no. 5, 1047-1061.

[2] Martn Argerami, Samuel Coskey, Mehrdad Kalantar, Matthew Kennedy, Martino Lupini, and Marcin Sabok, The classification problem for finitely generated operator systems and spaces, arXiv:1411.0512 (2014).

[3] Michael F. Atiyah and Friedrich Hirzebruch, Riemann-Roch theorems for differentiable manifolds, Bulletin of the American Mathematical Society 65 (1959), no. 4, 276-281.

[4] Selçuk Barlak and Gabór Szabó, Rokhlin actions of finite groups on UHF-absorbing $C^{*}$ algebras, arXiv:1403.7312 (2014).

[5] Itaï Ben Yaacov, Alexander Berenstein, C. Ward Henson, and Alexander Usvyatsov, Model theory for metric structures, Model theory with applications to algebra and analysis. Vol. 2, London Mathematical Society Lecture Note Series, vol. 350, Cambridge University Press, 2008, p. 315-427.

[6] Itaï Ben Yaacov, Andre Nies, and Todor Tsankov, A Lopez-Escobar theorem for continuous logic, arXiv:1407.7102 (2014).

[7] Bruce Blackadar, K-theory for operator algebras, Mathematical Sciences Research Institute Publications, vol. 5, Springer-Verlag, New York, 1986.

[8] _ Operator algebras, Encyclopaedia of Mathematical Sciences, vol. 122, SpringerVerlag, Berlin, 2006.

[9] Joan Bosa, Nathanial P. Brown, Yasuhiko Sako, Aaron Tikuisis, Stuart White, and Wilhelm Winter, Covering dimension of $C^{*}$-algebras and the classification of maps by traces, in preparation.

[10] Ola Bratteli, Inductive limits of finite dimensional $C^{*}$-algebras, Transactions of the American Mathematical Society 171 (1972), 195-234. 
[11] Nathanial P. Brown and Narutaka Ozawa, $C^{*}$-algebras and finite-dimensional approximations, Graduate Studies in Mathematics, vol. 88, American Mathematical Society, 2008.

[12] Riccardo Camerlo and Su Gao, The completeness of the isomorphism relation for countable Boolean algebras, Transactions of the American Mathematical Society 353 (2001), no. 2, 491-518.

[13] Samuel Coskey and Martino Lupini, A López-Escobar theorem for metric structures, and the topological Vaught conjecture, arXiv:1405.2859 (2014).

[14] Joachim Cuntz, Simple $C^{*}$-algebras generated by isometries, Communications in Mathematical Physics 57 (1977), no. 2, 173-185.

[15] _ K-theory for certain $C^{*}$-algebras. II, Journal of Operator Theory 5 (1981), no. 1, $101-108$.

[16] Kenneth R. Davidson, $C^{*}$-algebras by example, Fields Institute Monographs, vol. 6, American Mathematical Society, 1996.

[17] Rod Downey and Antonio Montalbán, The isomorphism problem for torsion-free abelian groups is analytic complete, Journal of Algebra 320 (2008), no. 6, 2291-2300.

[18] Christopher J. Eagle, Ilijas Farah, Bradd Hart, Boris Kadets, Vladyslav Kalashnyk, and Martino Lupini, Fraïssé limits of $C^{*}$-algebras, arXiv:1411.4066 (2014).

[19] Edward G. Effros, Some quantizations and reflections inspired by the Gelfand-Naimark theorem, C*-algebras: 1943-1993 (San Antonio, TX, 1993), Contemporary Mathematics, vol. 167, Amer. Math. Soc., Providence, RI, 1994, pp. 98-113.

[20] George A Elliott, On the classification of inductive limits of sequences of semisimple finitedimensional algebras, Journal of Algebra 38 (1976), no. 1, 29-44.

[21] George A. Elliott, Some $C^{*}$-algebras with outer derivations, III, Annals of Mathematics 106 (1977), no. 1, 121-143.

[22] - The classification problem for amenable $C^{*}$-algebras, Proceedings of the International Congress of Mathematicians, Vol. 1, 2 (Zürich, 1994), Birkhäuser, 1995, p. 922-932.

[23] George A. Elliott, Ilijas Farah, Vern Paulsen, Christian Rosendal, Andrew S. Toms, and Asger Törnquist, The isomorphism relation for separable $C^{*}$-algebras, Mathematical Research Letters 20 (2013), no. 6, 1071-1080.

[24] George A. Elliott and Andrew S. Toms, Regularity properties in the classification program for separable amenable $C^{*}$-algebras, Bulletin of the American Mathematical Society 45 (2008), no. 2, 229-245.

[25] Ilijas Farah, A dichotomy for the Mackey Borel structure, Proceedings of the 11th Asian Logic Conference, World Sci. Publ., Hackensack, NJ, 2012, p. 86-93.

[26] _ Logic and operator algebras, Proceedings of the International Congress of Mathematicians (Seoul, South Corea), 2014.

[27] Ilijas Farah, Bradd Hart, Martino Lupini, Leonel Robert, Aaron P. Tikuisis, Alessandro Vignati, and Wilhelm Winter, Model theory of nuclear $C^{*}$-algebras, in preparation.

[28] Ilijas Farah, Bradd Hart, and David Sherman, Model theory of operator algebras II: Model theory, Israel Journal of Mathematics, to appear.

[29] Ilijas Farah, Andrew S. Toms, and Asger Törnquist, The descriptive set theory of $C^{*}$-algebra invariants, International Mathematics Research Notices (2012), 5196-5226.

[30] _ Turbulence, orbit equivalence, and the classification of nuclear $C^{*}$-algebras, Journal für die reine und angewandte Mathematik 688 (2014), 101-146. 
[31] Valentin Ferenczi, Alain Louveau, and Christian Rosendal, The complexity of classifying separable banach spaces up to isomorphism, Journal of the London Mathematical Society 79 (2009), no. 2, 323-345.

[32] Matthew Foreman and Benjamin Weiss, An anti-classification theorem for ergodic measure preserving transformations, Journal of the European Mathematical Society 6 (2004), no. 3, 277-292.

[33] Harvey Friedman and Lee Stanley, A borel reductibility theory for classes of countable structures, Journal of Symbolic Logic 54 (1989), no. 03, 894-914.

[34] Su Gao, Invariant descriptive set theory, Pure and Applied Mathematics (Boca Raton), vol. 293, CRC Press, Boca Raton, FL, 2009.

[35] Su Gao and Alexander S. Kechris, On the classification of polish metric spaces up to isometry, Memoirs of the American Mathematical Society 161 (2003), no. 766, 0-0.

[36] Eusebio Gardella and Martino Lupini, Conjugacy and cocycle conjugacy of automorphisms of $\mathcal{O}_{2}$ are not Borel, arXiv:1404.3617 (2014).

[37] Izrail M. Gelfand and Mark A. Neumark, On the imbedding of normed rings into the ring of operators in Hilbert space, Matematicheskii Sbornik Novaya Seriya 12(54) (1943), 197-213.

[38] James Glimm, Type I $C^{*}$-algebras, Annals of Mathematics. Second Series 73 (1961), 572612.

[39] Leo A. Harrington, Alexander S. Kechris, and Alain Louveau, A Glimm-Effros dichotomy for Borel equivalence relations, Journal of the American Mathematical Society 3 (1990), no. 4, 903-928.

[40] Joseph Harrison, Recursive pseudo-well-orderings, Transactions of the American Mathematical Society 131 (1968), 526-543.

[41] Greg Hjorth, Non-smooth infinite-dimensional group representations, preprint, 1997.

[42] _ Classification and orbit equivalence relations, Mathematical Surveys and Monographs, vol. 75, American Mathematical Society, Providence, RI, 2000.

[43] _ On invariants for measure preserving transformations, Fundamenta Mathematicae 169 (2001), no. 1, 51-84.

[44] _ The isomorphism relation on countable torsion free abelian groups, Fundamenta Mathematicae 175 (2002), no. 3, 241-257.

[45] Greg Hjorth and Alexander S. Kechris, New dichotomies for Borel equivalence relations, The Bulletin of Symbolic Logic 3 (1997), no. 3, 329-346.

[46] Masaki Izumi, Finite group actions on $C^{*}$-algebras with the Rohlin property, I, Duke Mathematical Journal 122 (2004), no. 2, 233-280.

[47] _ Finite group actions on $C^{*}$-algebras with the Rohlin property - II, Advances in Mathematics 184 (2004), no. 1, 119-160.

[48] Xinhui Jiang and Hongbing $\mathrm{Su}$, On a simple unital projectionless $C^{*}$-algebra, American Journal of Mathematics 121 (1999), no. 2, 359-413.

[49] Alexander S. Kechris, Classical descriptive set theory, Graduate Texts in Mathematics, vol. 156, Springer-Verlag, New York, 1995.

[50] _ New directions in descriptive set theory, The Bulletin of Symbolic Logic 5 (1999), no. $2,161-174$.

[51] _ Global aspects of ergodic group actions, Mathematical Surveys and Monographs, vol. 160, American Mathematical Society, Providence, RI, 2010. 
[52] Alexander S. Kechris and Nikolaos E. Sofronidis, A strong generic ergodicity property of unitary and self-adjoint operators, Ergodic Theory and Dynamical Systems 21 (2001), no. 5, 1459-1479.

[53] David Kerr, Hanfeng Li, and Mikaël Pichot, Turbulence, representations, and tracepreserving actions, Proceedings of the London Mathematical Society 100 (2010), no. 2, 459-484.

[54] David Kerr, Martino Lupini, and N. Christopher Phillips, Borel complexity and automorphisms of $C^{*}$-algebras, accepted for publication by the Journal of Functional Analysis.

[55] Eberhard Kirchberg, Exact $C^{*}$-algebras, tensor products, and the classification of purely infinite algebras, Proceedings of the International Congress of Mathematicians, Vol. 1, 2 (Zürich, 1994), Birkhäuser, Basel, 1995, p. 943-954.

[56] Eberhard Kirchberg and N. Christopher Phillips, Embedding of exact $C^{*}$-algebras in the Cuntz algebra $\mathcal{O}_{2}$, Journal für die reine und angewandte Mathematik 525 (2000), 17-53.

[57] Akitaka Kishimoto, The Rohlin property for automorphisms of UHF algebras, Journal für die reine und angewandte Mathematik 1995 (1995), no. 465, 183-196.

[58] Elon Lindenstrauss, Mean dimension, small entropy factors and an embedding theorem, Institut des Hautes tudes Scientifiques. Publications Mathmatiques (1999), no. 89, 227-262 (2000).

[59] Elon Lindenstrauss and Benjamin Weiss, Mean topological dimension, Israel Journal of Mathematics 115 (2000), 1-24.

[60] Alain Louveau and Christian Rosendal, Complete analytic equivalence relations, Transactions of the American Mathematical Society 357 (2005), no. 12, 4839-4866.

[61] Martino Lupini, Polish groupoids and functorial complexity, arXiv:1407.6671 (2014).

[62] _ Unitary equivalence of automorphisms of separable $C^{*}$-algebras, Advances in Mathematics 262 (2014), 1002-1034.

[63] George W. Mackey, Borel structure in groups and their duals, Transactions of the American Mathematical Society 85 (1957), no. 1, 134-165.

[64] Julien Melleray, Computing the complexity of the relation of isometry between separable banach spaces, Mathematical Logic Quarterly 53 (2007), no. 2, 128-131.

[65] Benjamin D. Miller, The graph-theoretic approach to descriptive set theory, Bulletin of Symbolic Logic 18 (2012), no. 4, 554-575.

[66] Francis J. Murray and John von Neumann, On rings of operators. IV, Annals of Mathematics 44 (1943), no. 4, 716-808.

[67] Hideki Nakamura, Aperiodic automorphisms of nuclear purely infinite simple $C^{*}$-algebras, Ergodic Theory and Dynamical Systems 20 (2000), no. 6, 1749-1765.

[68] Narutaka Ozawa and Gilles Pisier, A continuum of $c^{*}$-norms on $\mathbb{B}(H) \otimes \mathbb{B}(H)$ and related tensor products, to appear.

[69] John Phillips, Outer automorphisms of separable $C^{*}$-algebras, Journal of Functional Analysis 70 (1987), no. 1, 111-116.

[70] John Phillips and Iain Raeburn, Automorphisms of $C^{*}$-algebras and second Čech cohomology, Indiana University Mathematics Journal 29 (1980), no. 6, 799-822.

[71] N. Christopher Phillips, The tracial Rokhlin property is generic, arXiv:1209.3859 (2012).

[72] Sorin Popa, Deformation and rigidity for group actions and von Neumann algebras, International Congress of Mathematicians. Vol. I, European Mathematical Society, Zrich, 2007, pp. $445-477$. 
[73] Mikael Rørdam, A simple $C^{*}$-algebra with a finite and an infinite projection, Acta Mathematica 191 (2003), no. 1, 109-142.

[74] Jonathan Rosenberg, Algebraic K-theory and its applications, Graduate Texts in Mathematics, vol. 147, Springer-Verlag, New York, 1994.

[75] Christian Rosendal, The generic isometry and measure preserving homeomorphism are conjugate to their powers, Fundamenta Mathematicae 205 (2009), no. 1, 1-27.

[76] Marcin Sabok, Completeness of the isomorphism problem for separable $C^{*}$-algebras, arXiv:1306.1049 (2013).

[77] Yasuhiko Sato, The Rohlin property for automorphisms of the Jiang-Su algebra, Journal of Functional Analysis 259 (2010), no. 2, 453-476.

[78] Claude Schochet, Algebraic topology and $C^{*}$-algebras, C*-algebras: 1943-1993 (San Antonio, TX, 1993), Contemporary Mathematics, vol. 167, Amer. Math. Soc., Providence, RI, 1994, pp. 218-231.

[79] Irving Segal, $C^{*}$-algebras and quantization, C*-algebras: 1943-1993 (San Antonio, TX, 1993), Contemporary Mathematics, vol. 167, Amer. Math. Soc., Providence, RI, 1994, pp. 54-65.

[80] Jack H. Silver, Counting the number of equivalence classes of Borel and coanalytic equivalence relations, Annals of Mathematical Logic 18 (1980), no. 1, 1-28.

[81] Andrew S. Toms, On the independence of K-theory and stable rank for simple $C^{*}$-algebras, Journal für die reine und angewandte Mathematik 578 (2005), 185-199.

[82] _ On the classification problem for nuclear $C^{*}$-algebras, Annals of Mathematics 167 (2008), no. 3, 1029-1044.

[83] Comparison theory and smooth minimal $C^{*}$-dynamics, Communications in Mathematical Physics 289 (2009), no. 2, 401-433.

[84] Andrew S. Toms and Wilhelm Winter, Strongly self-absorbing $C^{*}$-algebras, Transactions of the American Mathematical Society 359 (2007), no. 8, 3999-4029.

[85] Simon Wassermann, On tensor products of certain group $C^{*}$-algebras, Journal of Functional Analysis 23 (1976), no. 3, 239-254.

[86] Matthew Wiersma, $C^{*}$-norms for tensor products of discrete group $C^{*}$-algebras, arXiv:1406.2654 (2014).

[87] Jindřich Zapletal, Forcing Borel reducibility invariants, in preparation.

[88] _ Analytic equivalence relations and the forcing method, Bulletin of Symbolic Logic 19 (2013), no. 4, 473-490.

[89] Joseph Zielinski, The complexity of the homeomorphism relation between compact metric spaces, arXiv:1409.5523 (2014).

Martino Lupini, Department of Mathematics and Statistics, N520 Ross, 4700 Keele Street, Toronto Ontario M3J 1P3, Canada, and Fields Institute for Research in Mathematical Sciences, 222 College Street, Toronto ON M5T 3J1, Canada.

E-mail address: mlupini@mathstat.yorku.ca

URL: http://www. lupini.org/ 